\begin{tabular}{|l|c|c|}
\hline \multicolumn{2}{|c|}{ DJS Vol. 38 (2017) 19-26 } \\
\hline 1969 & Delta Journal of Science & \\
\hline Research Article & Available online at & \\
\hline
\end{tabular}

\title{
Rough Convexity of A Set and A Function
}

\author{
E.A.Youness $^{1}$, A.A.ALsaraireh ${ }^{2}$, H.S.Alrawashdeh ${ }^{2}$ \\ 1- Faculty of science, Tanta University, Tanta ,Egypt \\ ebrahimyouness@science.tanta.edu.eg \\ 2 - Faculty of information Technology and systems, The University of Jordan ,Aqaba,Jordan \\ ah_8545@yahoo.com, a.alsarairah@ju.edu.jo \\ Faculty of information Technology and systems ,The University of Jordan ,Aqaba,Jordan \\ hsrawashdeh@yahoo.com
}

\begin{abstract}
In this paper a new concept of convexity approximation for non convex set and nonconvex function with respect to family of convex sets and to family of convex function respectively is presented. This approximation of convexity is called rough convexity. Some properties of these kind of sets and functions are discussed.
\end{abstract}

Key words: Rough convexity with respect to family of convex sets, rough supporting hyperplane, rough convex function with respect to family of convex functions.

\section{1 -Introduction}

The concept of convexity of sets and functions plays an important role in the field of mathematical programming. The convexity ensure the globality of solutions. In real life problems the convexity maybe not satisfied, so the objective function or the constraints or both may not be convex that pushes the researchers to relax non convexity of sets and functions to what is called generalized convexity such as, quasi, pseudo, E-convexity.

Recently E.A.Youness [ 3 ] discussed an optimization problem that involves roughness notion in the constraint set. Also Fang D., [ 6 ] presented rough approximation of non convex set.

In this paper the rough approximation non convex set and non convex function in terms of family of convex sets and family of convex functions, are presented and some their properties are discussed.

\section{2 - Rough convex set}

Definition 2.1 : Let $\mathrm{X}$ be an universal set, $\mathrm{F}$ be a family of non-disjoint convex sets

$$
F=\left\{A_{1}, A_{2}, \ldots, A_{n}\right\} \quad, B \subset X \text { and } \sigma(x, y) \text { is }
$$

a space of segments between $\mathrm{x}$ and $\mathrm{y}$ in $\mathrm{B}$. B is called rough convex with respect to family $\mathrm{F}$ if for each

$x, y \in B, \sigma(x, y)$ either contained in $\bigcap_{i=1}^{n} A_{i}$

or $\sigma \bigcap A_{i} \neq \varphi$ for at least one i .

The Lower convexity of $\mathrm{B}$ is:

$L(\operatorname{Conv})=\left\{\sigma(x, y): \sigma(x, y) \subset \bigcap_{i=1}^{n} A_{i}, \sigma(x, y) \subset B\right\}$

and

The upper convexity of B is: 
$U($ Conv $)=\left\{\sigma(x, y): \sigma(x, y) \cap A_{i} \neq \phi\right.$ for at least one $\left.i\right\}$

The following figures show the rough convexity of a set $\mathrm{B}$ with respect to a family of a convex sets.

In figure $(2-1)$ and figure $(2-2)$ the sets $\mathrm{B}$ and $\mathrm{B}^{\prime}$ are rough convex with respect to $A_{1}, A_{2}$ and $A_{3}$, but in figure $(2-3)$ and figure $(2-4)$ the sets $\mathrm{B}$ " and B"' are non rough convex with respect to them.

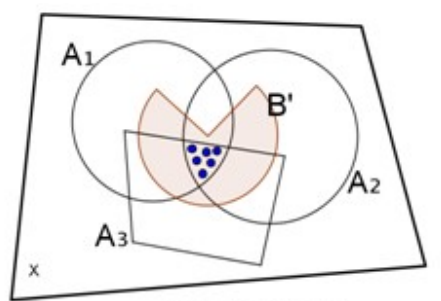

Figure (2-1)

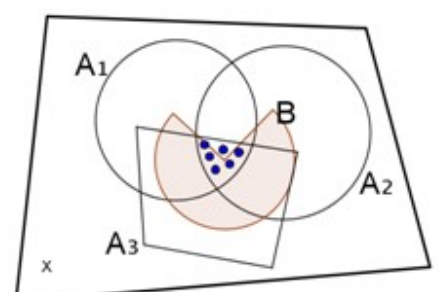

Figure (2-2)

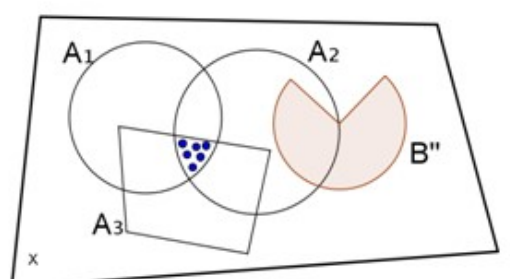

Figure (2-3)

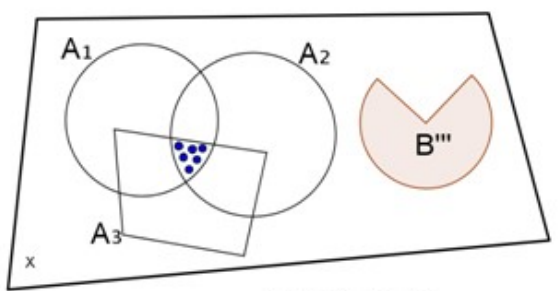

Figure (2-4)

Example 2.1 : Consider a universal

$$
X=\left\{(x, y) \in R^{2}:-3 \leq x \leq 3 \quad, \quad-3 \leq y \leq 3\right\}
$$

and family F :

$$
F=\left\{\begin{array}{l}
A_{1}=\left\{(x, y) \in R^{2}:(x)^{2}+(y+1)^{2} \leq 2\right\} \\
A_{2}=\left\{(x, y) \in R^{2}:(x-1)^{2}+(y-1)^{2} \leq 3\right\} \\
A_{3}=\left\{(x, y) \in R^{2}:(x+1)^{2}+(y-1)^{2} \leq 3\right\}
\end{array}\right\}
$$

A set

$B=\left\{(x, y) \in R^{2}: y \geq \frac{1}{2} x^{2}, \quad y \leq|0.5 x|+1, \quad-2 \leq x \leq 2 \quad\right\}$ is rough convex with respect to $\mathrm{F}$ with:

Lower convexity of

$$
L=\left\{\sigma: \sigma=\lambda x+(1-\lambda) y, 0 \leq \lambda \leq 1 x, y \in \bigcap_{i=1}^{3} A_{i}\right\}
$$

\section{And}

upper convexity

$U=\{\sigma: \sigma=\lambda x+(1-\lambda) y, 0 \leq \lambda \leq 1 x, y \in B\}$, see Figure (2-5).

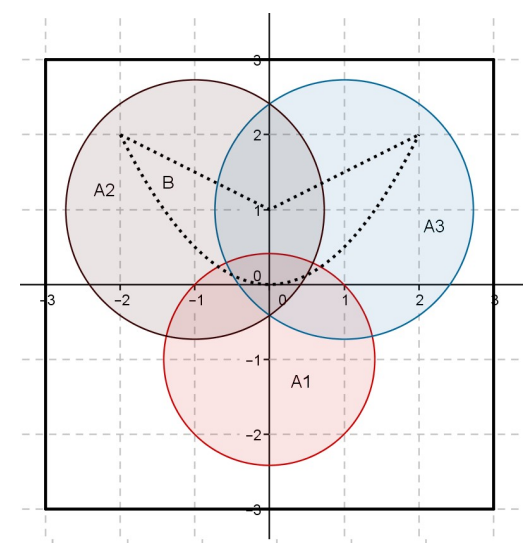

Figure ( 2 - 5 )

Remark 2.1 :.If $\mathbf{L}$ (conv) $=\mathbf{U}($ conv $)$ of the set $\mathbf{B}$ ,with respect to the family $F=\left\{A_{1}, A_{2}, \ldots, A_{n}\right\} \quad$,then the set $\mathrm{B}$ is completely convex and hence $B \subset \bigcap_{i=1}^{n} A_{i}$.

A convex set is rough convex with respect to itself and its $\mathbf{L}(\mathbf{c o n v})=$ its $\mathbf{U}(\mathbf{c o n v})$

Theorem 2.1: If $\mathbf{B}$ is a roughly convex with respect to a family of convex sets $A_{i}, i=1,2,3, \ldots, n$, then $B^{\prime}=f(B) \quad$ is a roughly

convex with respect to family $C_{i}, i=1,2,3, \ldots, n$ ,such that $f\left(x_{C i}\right)=x_{A i} \mp \alpha, x_{C i} \in C_{i}$ where 
$x_{c i} \in C_{i}, x_{A i} \in A_{i} \quad$,and $\mathbf{B}^{\prime}$ is the image of

B (i.e : $\left.f(B)=B^{\prime}\right)$ ).

Proof: $\mathbf{B}$ is roughly convex with respect to $A_{i}$, so

$$
\sigma(x, y) \subset \bigcap_{i=1}^{n} A_{i} \quad \text { or } \quad \sigma(x, y) \bigcap A_{i} \neq \varphi \quad \text {, for }
$$

all $x, y \in B$.

uppose $x, y \in B^{\prime}=f(B), x, y \in \cup C_{i}$ thus, $f\left(x_{i}\right)=x_{A_{I}}+\alpha \quad, f\left(y_{C_{i}}\right)=y_{A_{i}}+\alpha$, and

$$
\begin{aligned}
\sigma\left(x_{c i}, y_{c i}\right) & =\lambda x_{C i}+(1-\lambda) y_{C i} \\
& =\lambda\left[x_{A i}+\alpha\right]+(1-\lambda)\left[y_{A i}+\alpha\right] \\
& =\sigma\left(x_{A i}, y_{A i}\right)+\alpha
\end{aligned}
$$

So,

$$
\sigma\left(x_{C_{i}}, y_{C_{i}}\right) \subset \bigcap_{i=1}^{n} C_{i} \quad \text { or } \quad \sigma\left(x_{C_{i}}, y_{C_{i}}\right) \cap C_{i} \neq \varphi
$$

. Hence $B^{\prime}=f(B)$ is roughly convex with respect to $C_{i}$

Theorem 2.2 : If $B \subset X$ is a roughly convex with respect to a family of convex sets $A_{i}, i=1,2,3, \ldots, n$.Let $f: X \rightarrow X$ be a map such that $f\left(A_{i}\right)=A_{j}, i, j \in\{1,2,3, \ldots \ldots, n\}$ then,$\quad B^{\prime}=f(B)$ is a roughly convex with respect to

family $A_{i}$.

Proof : The proof is clear, since for $\mathrm{x}^{\prime}, y^{\prime} \in B^{\prime}$, then there exist $\mathrm{x}, y \in B$ such that $\lambda \mathrm{x}^{\prime}+(1-\lambda) y^{\prime}=\lambda f(x)+(1-\lambda) f(y) \subset \bigcap_{i=1}^{n} A_{i}$ or $[\lambda f(x)+(1-\lambda) \mathrm{f}(\mathrm{y})] \cap A_{i} \neq \phi$, for some i

Example 2.2 : Consider the following two families of convex sets, $A=\left\{A_{1}, A_{2}, A_{3}\right\} \subset X \subset R \quad$ and $C=\left\{C_{1}, C_{2}, C_{3}\right\}$, and the relation between the two families is given by the following $f: A_{i} \rightarrow C_{i}$ such that

$f(x, y)=(x-6, y-2)$

$$
\begin{gathered}
X=\left\{(x, y) \in R^{2}:-4 \leq x \leq 9,-4 \leq y \leq 5\right\} \\
A=\left\{\begin{array}{l}
A_{1}=\left\{(x, y):(x+1)^{2}+(y+1)^{2} \leq 4\right. \\
A_{2}=\left\{(x, y):(x)^{2}+(y-1)^{2} \leq 2\right. \\
A_{3}=\left\{(x, y):(x-1)^{2}+(y)^{2} \leq 3\right.
\end{array}\right\}
\end{gathered}
$$

The set

$B=\left\{x, y \in R^{2}: 5(x-0.3)^{2}-1, \quad|0.5(x-0.3)|+0.7, \quad-0.34 \leq x \leq 0.94\right\}$ is rough convex with respect to $\boldsymbol{A}$

$$
C=\left\{\begin{array}{l}
C_{1}=\left\{(x, y):(x-5)^{2}+(y-1)^{2} \leq 4\right\} \\
C_{2}=\left\{(x, y):(x-6)^{2}+(y-3)^{2} \leq 2\right\} \\
C_{3}=\left\{(x, y):(x-7)^{2}+(y-2)^{2} \leq 3\right\}
\end{array}\right\}
$$

and the set

$$
B^{\prime}=\left\{x, y \in R^{2}: 5(x-6.3)^{2}+1, \quad|0.5(x-6.3)|+2.7, \quad 5.66 \leq x \leq 6.94 \quad\right\}
$$
is rough convex with respect to $\boldsymbol{C}$,See Figure(2-6).

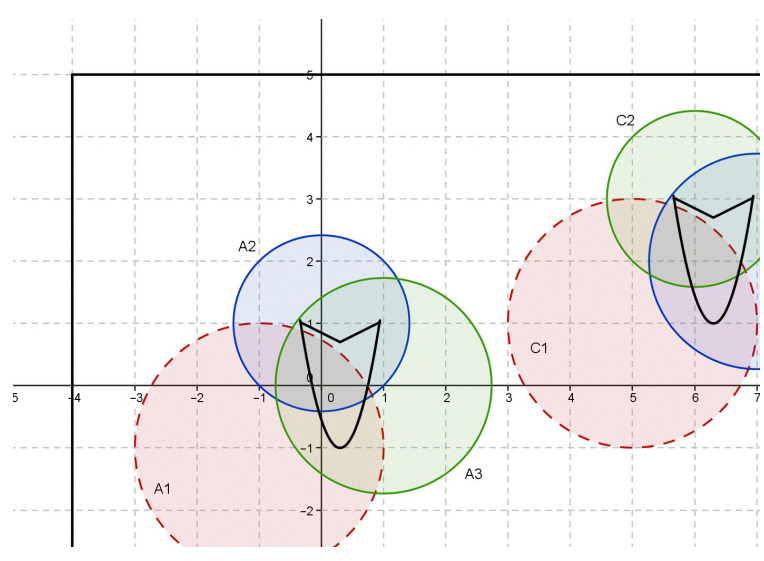

Figure ( 2-6).

Theorem 2.3 : Let $\mathrm{X}$ be an universal set,

$F=\left\{A_{1}, A_{2}, \ldots, A_{n}\right\} \quad$ is a family of convex subsets

of $\mathrm{X}$.If $B$ and $B^{\prime}$ are roughly convex with respect to $F=\left\{A_{1}, A_{2}, \ldots, A_{n}\right\}$, then

1. $B \cap B^{\prime}$ is a rough convex with respect to a family $\mathrm{F}$.

2. $B \cup B^{\prime}$ is not necessarily to be a rough convex with respect to a family $\mathrm{F}$.

Proof :

1. Let $x, y \in B \cap B^{\prime} \Rightarrow x, y \in B$ and $x, y \in B^{\prime}$. 
Assume $\sigma(x, y)$ the segment whose ends

$x$ and $y$.

From the roughly convexity of B and B' with respect to $F=\left\{A_{1}, A_{2}, \ldots, A_{n}\right\}$.

we have

$\sigma(x, y) \subset \bigcap A_{i} \quad$ or $\quad \sigma(x, y) \bigcap A_{i} \neq \varphi$, for at

least one $\mathrm{i}$.

So, $B \cap B^{\prime}$ is rough convexity with respect to a family of convex sets.

2. Let $x, y \in B \cup B^{\prime}$,

So may

$x, y \in B, x, y \in B^{\prime}$ or $x, y \in B \cap B^{\prime}$

Thus

$\sigma(x, y) \subset \bigcap A_{i}$ or $\sigma(x, y) \bigcap A_{i} \neq \varphi$, for at least one $i$

$\Rightarrow B \cup B^{\prime}$ is rough convexity but, If $x \in B$ and $y \in B^{\prime}$ implies that $B \cup B^{\prime}$ does not necessarily contain $\sigma$, see Figure (2-7)

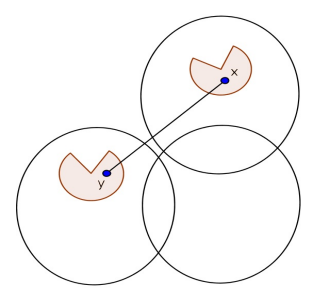

Figure ( 2-7)

Remark 2.2 :

The lower and upper approximation of

convexity for intersection and union of two roughly convex sets $\mathrm{B}$ and $\mathrm{B}^{\prime}$ given as:

$\left\langle\begin{array}{l}L_{\cap}(\text { Con })=\left\{\sigma(x, y): \sigma(x, y) \subset \bigcap_{:=1}^{n} A_{i}, \sigma(x, y) \in B \cap B^{\prime}\right\} \\ U_{\cap}(\text { Con })=\left\{\sigma(x, y): \sigma(x, y) \cap\left(\bigcap_{:=1}^{n} A_{i}\right) \neq \varphi, \text { for at least one } i\right\}\end{array}\right\rangle$ see Figure (2-8)

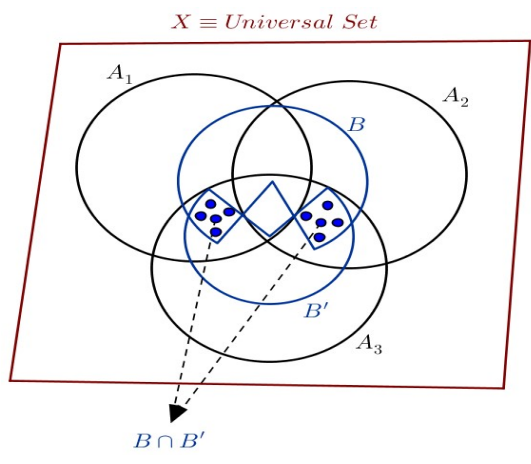

Figure ( 2-8 )

$\left\langle\begin{array}{l}L_{\cup}(\text { Con })=\left\{\sigma(x, y): \sigma(x, y) \subset \bigcap_{:=1}^{n} A_{i}, \sigma(x, y) \in B \cup B^{\prime}\right\} \\ U_{\cup}(\text { Con })=\left\{\sigma(x, y): \sigma(x, y) \cap\left(\bigcap_{:=1}^{n} A_{i}\right) \neq \varphi, \text { for at least one } i\right\}\end{array}\right\rangle$ see Figure ( 2-9)

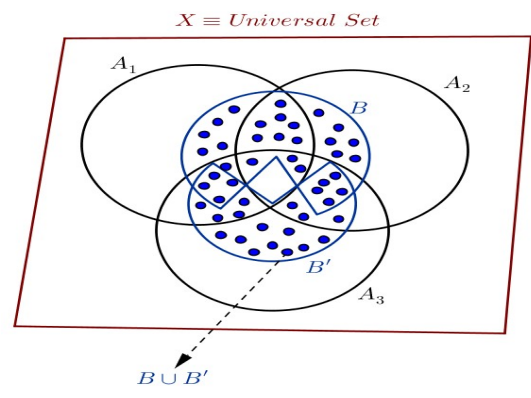

Figure (2-9)

Preposition 2.1 : Let $\mathrm{X}$ be an universal set, and $B$ and $B^{\prime}$ are roughly convex with respect to a family of convex sets $F=\left\{A_{1}, A_{2}, \ldots, A_{n}\right\}$, then the following properties are satisfying

\section{Intersection properties}

$L(\operatorname{con} v)_{B \cap B^{\prime}} \subset L(\operatorname{con} v)_{B} \cup L(\operatorname{con} v)_{B^{\prime}}$

( always true ) .

$U(\operatorname{con} v)_{B \cap B^{\prime}} \subset U(\operatorname{con} v)_{B} \cup U(\operatorname{con} v)_{B^{\prime}}$

( always true ) .

2. Union properties

$L(\operatorname{con} v)_{B \cup B^{\prime}} \supset L(\operatorname{con} v)_{B} \quad, L(\operatorname{con} v)_{B^{\prime}}$

( always true ).

$U(\operatorname{con} v)_{B \cup B^{\prime}} \supset U(\operatorname{con} v)_{B}, U(\operatorname{con} v)_{B^{\prime}}$

\section{( not always true ).}

\section{Proof :}

1. Intuitively

$B \cap B^{\prime} \subset B$ and $B \cap B^{\prime} \subset B^{\prime}$

If

$\sigma(x, y) \subset B \cap B^{\prime} \Rightarrow \sigma(x, y) \subset B$ and $\sigma(x, y) \subset B^{\prime}$

So, $\quad L(\operatorname{con} v)_{B \cap B^{\prime}} \subset L(\operatorname{con} v)_{B} \cup L(\operatorname{con} v)_{B^{\prime}}$, and

$U(\operatorname{con} v)_{B \cap B^{\prime}} \subset U(\operatorname{con} v)_{B} \cup U(\operatorname{con} v)_{B}{ }^{\prime}$

2. Since $B \cup B^{\prime} \supset B$ and $B \cup B^{\prime} \supset B^{\prime}$ 
If

$\sigma(x, y) \subset B, \sigma(x, y) \subset B^{\prime}$ or $\sigma(x, y) \subset B \cap B^{\prime}$ then

$\sigma(x, y) \subset B \cup B^{\prime}$

So,

$L(\operatorname{con} v)_{B \cup B}, \supset L(\operatorname{con} v)_{B}$ and $L(\operatorname{con} v)_{B}$,

$U(\operatorname{con} v)_{B \cup B^{\prime}} \supset U(\operatorname{con} v)_{B}$ and $U(\operatorname{con} v)_{B^{\prime}}$

But

$U(\operatorname{con} v)_{B \cup B^{\prime}} \supset U(\operatorname{con} v)_{B}$ and $U(\operatorname{con} v)_{B^{\prime}}$

is not necessary to be true when $x \in B$ and $y \in B^{\prime}$.

\section{3 - Rough Supporting Hyperplane}

Definition 3.1 : Let $\mathrm{X}$ be an universal set in $R^{n}$ ,B is a rough convex set with respect to family of convex sets $\mathfrak{I}=\left\{A_{1}, A_{2}, \ldots, A_{n}\right\}$, $H=\left\{\tilde{H}_{1}, \tilde{H}_{2}, \tilde{H}_{3}\right\} \subset \mathrm{H}$ is the set of all supporting hyperplanes of the family .Consider the following three classes :

$\tilde{H}_{1}=\left\{H: \begin{array}{l}H \text { is supporting for at least one } \\ \text { of the family and } H \cap B=\phi\end{array}\right\}$

$\tilde{H}_{2}=\left\{\begin{array}{c}H \text { is supporting for at least one of the } \\ \text { family and } H \cap B \neq \phi \text { at one point }\end{array}\right\}$

$\tilde{H}_{3}=\left\{H: \begin{array}{l}H \text { is supporting for at least one of the family } \\ \text { and } H \cap B \neq \phi \text { at least one point }\end{array}\right\}$

Each element $h \in H \subset \mathrm{H}$ is called rough supporting of $\mathrm{B}$, if $\mathrm{H}$ is a rough set in $\mathrm{H}$.

Upper Supporting approximation:

$U_{H}=\{H: H$ is supporting for at least one, of family and $H \cap B \neq \varphi\}$ , see Figure ( 3-1 ).

\section{Lower Supporting approximation:}

$L_{H}=\{H: H$ is supporting for at least one, of family and $H \cap B=\varphi\}$ , see Figure ( 3-2 ).
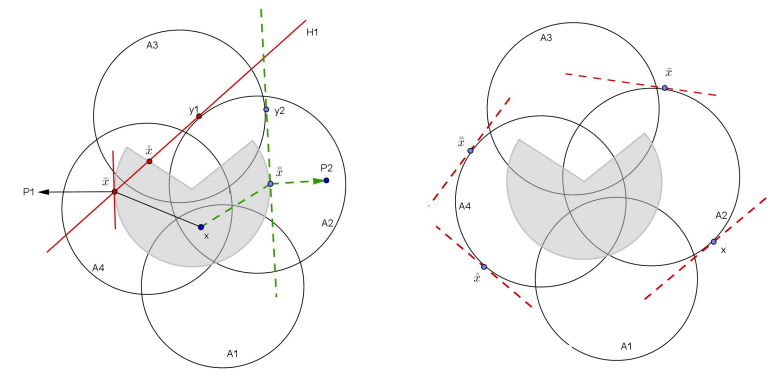

Figure ( 3-1)

Figure ( 3- 2 )

\section{4 - Rough Convexity of a Function}

\section{Definition 4.1 : Let}

$\mathfrak{I}=\left\{f_{i}: B \rightarrow R, i=1,2, . ., k, f_{i}(x)\right.$ convex $\}$

;

be a family of convex functions, $f(x)$ is called rough convex on a convex set $B \subset R^{n} \quad$ with respect to $\mathfrak{J}$, if for each $x, y \in B$, there exist $f_{l}(x), f_{t}(x) \in \mathfrak{J}$, such that

$f(\lambda x+(1-\lambda) y) \leq \lambda f_{l}(x)+(1-\lambda) f_{t}(y), \quad 0 \leq \lambda \leq 1$

The upper convexity of $\mathbf{f}$ is denoted by $U$ (conv $f$ )

and is defined as

$U(\operatorname{conv} f)=\left\{f_{i} \in \mathfrak{I}: f_{i}(\mathrm{z})>f(\mathrm{z}), \mathrm{z} \in \lambda \mathrm{x}+(1-\lambda) \mathrm{y}, i \in\{1, \ldots ., k\}\right\}$

The lower convexity of $\mathbf{f}$ is denoted by $L(\operatorname{con} v f)$

and is defined as

$L(\operatorname{conv} f)=\left\{f_{i} \in \mathfrak{I}: f_{i}(\mathrm{z}) \leq f(\mathrm{z}), \mathrm{z} \in \lambda \mathrm{x}+(1-\lambda) \mathrm{y}, i \in\{1, \ldots ., k\}\right\}$

It is clear that for each $f_{h} \in L(\operatorname{con} v f)$,

$\operatorname{implies} f_{h}(x)<f_{i}(x) \in U(\operatorname{conv} f)$.

Rough concavity it is defined in similar way by reversing the sign $\leq$ to $\geq$,i.e: $f(\lambda x+(1-\lambda) y) \geq \lambda f_{l}(x)+(1-\lambda) f_{t}(y) \quad, \quad 0 \leq \lambda \leq 1$

\section{Examples 4.1 :}

Let

$\mathfrak{I}=\left\{\begin{array}{l}f_{1}(x)=e^{-x}+2, f_{2}(x)=2 x^{2}-6 x+4, \\ f_{3}(x)=x^{2}-2, f_{4}(x)=e^{-x}+20, f_{5}(x)=x^{2}+16\end{array}\right\}$

be a family of convex function defined on

$B=[0,5]$. The function

$f(x)=\frac{1}{3} x^{3}-x^{2}-x+15$ be a rough convex

function on $\mathrm{B}$ with respect to a family of convex functions $\mathfrak{I}$. and its upper and lower convexity are

$$
\left\langle\begin{array}{l}
U(\operatorname{conv} f)=\left\{f_{4}(x), f_{5}(x)\right\} \\
L(\operatorname{conv} f)=\left\{f_{1}(\mathrm{x}), f_{3}(x)\right\}
\end{array}\right\rangle
$$

, see Figure ( 4-1 ) 


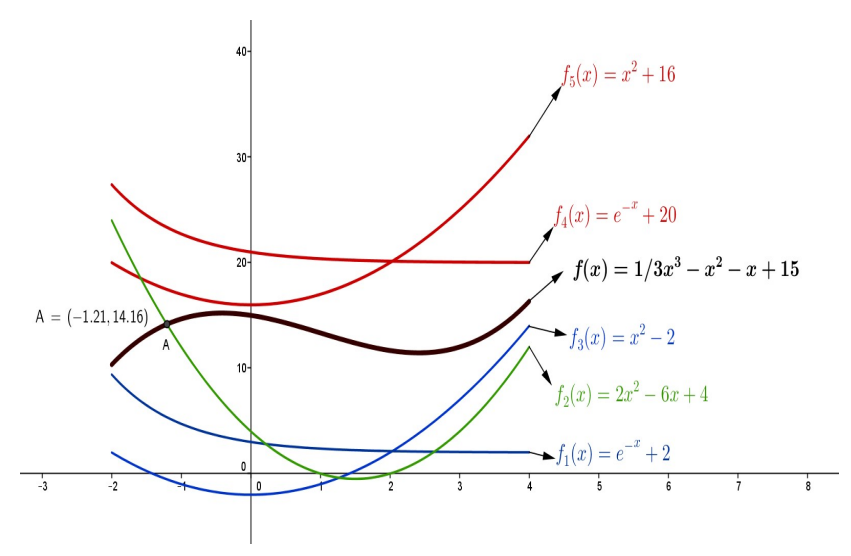

Figure ( 4-1)

In the following, the characterization of rough convexity of a function, in terms of rough convexity of its level set and its epigragh, is discussed .

\section{5 - Rough Epigraph}

Definition 5.1 : Let $E_{i}$ be an epigraph of a convex function $f_{i}$, let $f$ be a rough convex with respect to the family $f_{i}$.An epigraph of $f$ is denoted by

$E_{f}$ and is defined as : $E_{f}=\{(x, \alpha): f(x) \leq \alpha\}$.

Theorem 5.1 : If $f$ is a rough convex with respect to $f_{i}$, then $E_{f}$ is rough convex set with espect to $E_{i}$.

Proof: Assume $\left(x_{1}, y_{1}\right)$ and $\left(x_{2}, y_{2}\right) \in E_{f}$, i.e.,

$$
f\left(x_{1}\right) \leq y_{1}, f\left(x_{2}\right) \leq y_{2} .
$$

Since $\mathrm{f}$ is rough convex with respect to family $\mathfrak{I}$, so, there exist $f_{l}, f_{t} \in \mathfrak{I}$ such that

$f\left(\lambda x_{1}+(1-\lambda) x_{2}\right) \leq \lambda f_{l}\left(x_{1}\right)+(1-\lambda) f_{t}\left(x_{2}\right), \lambda \in[0,1]$

If $f_{l}$ and $f_{t}$ are in $L(\operatorname{conv} f)$, then

$f_{l}\left(x_{1}\right) \leq f\left(x_{1}\right) \leq y_{1}$ and

$f_{t}\left(x_{2}\right) \leq f\left(x_{2}\right) \leq y_{2}$. Therefore,

$f\left(\lambda x_{1}+(1-\lambda) x_{2}\right) \leq \lambda y_{1}+(1-\lambda) y_{2}$.

Since $f_{l}$ and $f_{t}$ are convex, so if

$$
f_{t}\left(x_{1}\right) \leq f_{t}\left(x_{2}\right) \leq y_{2}
$$

then $\left(\lambda x_{1}+(1-\lambda) x_{2}\right),\left(\lambda y_{1}+(1-\lambda) y_{2}\right) \in E_{l}\left(f_{l}\right)$

Similarly

$\left(\lambda x_{1}+(1-\lambda) x_{2}\right),\left(\lambda y_{1}+(1-\lambda) y_{2}\right) \in E_{t}\left(f_{t}\right)$

which implies

$\left\{\left(\lambda x_{1}+(1-\lambda) x_{2}\right),\left(\lambda y_{1}+(1-\lambda) y_{2}\right)\right\} \cap E_{i} \neq \varphi$

for at least one $\mathrm{i}$.

Thus the rough convexity of $E(f)$ on the other hand, if $f_{l}$ or $f_{t} \in U(\operatorname{conv} f)$, then for $\left(x_{1}, \mathrm{y}_{1}\right),\left(\mathrm{x}_{2}, y_{2}\right) \in E_{i}\left(f_{i}\right)$, we get

$\left(x_{1}, \mathrm{y}_{1}\right),\left(\mathrm{x}_{2}, y_{2}\right) \in E(f)$.

Since $f_{l}$ is convex

$,\left(\lambda x_{1}+(1-\lambda) x_{2}\right),\left(\lambda y_{1}+(1-\lambda) y_{2}\right) \in E_{l}\left(f_{l}\right)$,

i.e., $f_{l}\left(\lambda x_{1}+(1-\lambda) x_{2}\right) \leq \lambda y_{1}+(1-\lambda) y_{2}$.

Thus

$f_{l} \in U(\operatorname{conv} f)$ implies to

$$
\begin{aligned}
f\left(\lambda x_{1}+(1-\lambda) x_{2}\right) & \leq f_{l}\left(\lambda x_{1}+(1-\lambda) x_{2}\right) . \\
& \leq \lambda y_{1}+(1-\lambda) y_{2}
\end{aligned}
$$

Hence the result .

Lemma 5.1 : Let $\mathrm{S}$ be a nonempty convex set subset in $R^{n}$, let $f_{1}, f_{2}$ be rough convex with respect to family of convex functions

$\mathfrak{I}=\left\{f_{i}(\mathrm{x}): R^{n} \rightarrow R, i=1,2, . ., n\right\} \quad$.If $\mathfrak{I}$ is closed under addition then $f_{1}(x)+f_{2}(x)$ is rough convex.

Proof: Let $\quad g(x)=f_{1}(x)+f_{2}(x)$, $f_{1}(x), f_{2}(x)$ are rough convex that means, for each $\quad x_{1}, x_{2} \in S, 0 \leq \lambda \leq 1$, there exist $f_{t}(\mathrm{x}), f_{l}(\mathrm{x}), f_{n}(\mathrm{x}), f_{m}(x) \in \mathfrak{I}$ such that :

$z=\lambda x_{1}+(1-\lambda) x_{2} \in S, 0 \leq \lambda \leq 1$ and from rough convexity of $f_{1}(x)$ and $f_{2}(x)$, We have

$f_{1}(z) \leq \lambda f_{t}\left(x_{1}\right)+(1-\lambda) f_{l}\left(x_{2}\right)$

and $f_{2}(z) \leq \lambda f_{n}\left(x_{1}\right)+(1-\lambda) f_{m}\left(x_{2}\right)$

which implies 


$$
\begin{aligned}
g(z) & =\left(f_{1}+f_{2}\right)(z)=f_{1}(z)+f_{2}(z) \\
& \leq \lambda f_{t}\left(x_{1}\right)+(1-\lambda) f_{l}\left(x_{2}\right)+\lambda f_{n}\left(x_{1}\right)+(1-\lambda) f_{m}\left(x_{2}\right) \\
& =\lambda\left[f_{t}\left(x_{1}\right)+f_{n}\left(x_{1}\right)\right]+(1-\lambda)\left[f_{l}\left(x_{2}\right)+f_{m}\left(x_{2}\right)\right] \\
& =\lambda f_{k}\left(x_{1}\right)+(1-\lambda) f_{r}\left(x_{2}\right), \text { where } \\
f_{k}= & f_{t}+f_{n}, f_{r}=f_{l}+f_{m}
\end{aligned}
$$

From closeness of $\mathfrak{I}$ under addition, $f_{k}, f_{r} \in \mathfrak{I}$. Hence the result.

corollary 5.1 : Let $\mathrm{S}$ be a nonempty convex set subset in $\mathrm{R}^{\mathrm{n}}$, let $f_{1}(x)$ be rough convex and $f_{2}(x)$ be rough concave with respect to family of convex functions

$\mathfrak{I}=\left\{f_{i}: R^{n} \rightarrow R, i=1,2, . ., n\right\}$. Then $f_{1}(x)-f_{2}(x)$ is rough convex.

Proof: The proof immediately yields from lemma 5.1

\section{6 - Level set}

Definition 6.1 : Let $S$ be a nonempty convex set subset in $\mathrm{R}^{\mathrm{n}}$. Let $f: S \rightarrow R$ be a rough convex with respect to family of convex functions $\mathfrak{J}=\left\{f_{i}: R^{n} \rightarrow R, i=1,2, . ., n\right\}$. A level set of $f(x)$ is defined as $S_{\alpha}=\{x \in S: f(x) \leq \alpha\}$, it is clear that $S_{\alpha} \subset \cup S_{\alpha_{i}} \quad, \quad$ where $S_{\alpha_{i}}=\left\{x \in S: f_{i}(x) \leq \alpha_{i}\right\}$. see Figure (6-1)

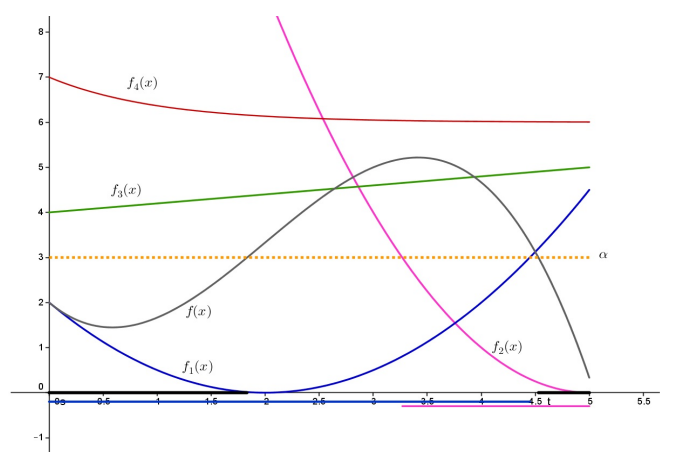

Figure ( 6-1)

Theorem 6.2 : Let $f$ be a rough convex with respect to family convex functions

$$
\mathfrak{I}=\left\{f_{i}: R^{n} \rightarrow R, i=1,2, . ., n\right\} \quad \text {. Assume }
$$

$S_{\alpha i}$ is the level set of $f_{i}$. A level set $S_{\alpha}$ of $f$ is rough convex with respect to $S_{\alpha_{i}}$, $\alpha=\max \alpha_{i}$.
Proof: Since $S_{\alpha} \subset \cup S_{\alpha_{i}}$ $x, y \in \cup S_{\alpha_{i}}$ then $x, y$ may be in $\cap S_{\alpha_{i}}$ which implies $\lambda x+(1-\lambda) y \subset \bigcap_{i} S_{\alpha_{i}}$

Thus $f_{i}(\lambda x+(1-\lambda) y) \leq \alpha_{i} \leq \alpha$ since $f$ is rough convex, there exist $f_{l}$ and $f_{t}$, such that

$f(\lambda x+(1-\lambda) y) \leq \lambda f_{l}(x)+(1-\lambda) f_{t}(y) \leq \lambda \alpha_{l}+(1-\lambda) \alpha_{t} \leq \alpha$ On the other hand, $x, y \notin \cap S_{\alpha_{i}}, x, y \in \bigcup S_{\alpha_{i}}$, $\{\lambda x+(1-\lambda) y\} \cap S_{\alpha_{i}} \neq \phi$ for at least one i .

\section{Conclusion :}

In this paper the definition of rough convexity of set with respect to a family of convex sets ,and rough convexity of function with respect to a family of convex functions . Therefore we discussed the relationship between roughness with respect to a family of functions and their epigraphs and level sets.

\section{References.}

E. A. Youness -E-Convex Sets, E-Convex Functions, and E-Convex Programming, Journal Of Optimization Theory And Applications: Vol. 102, No. 2, pp. 439450, August 1999.

E.A.Youness and Tarek Emam, Semi Strongly E-convex Functions, Journal of Mathematics and Statistics 1(2005) (1):5157.

E.A.Youness and Tarek Emam, Strongly Econvex Sets and Strongly Econvex Functions, Journal of Interdisciplinear Mathematics.Vol.8 (2005), No.1 pp.107$117,2005$.

F.A.Deng, On the rough approximation of non-convex set,J. of university of science and technology of suzhou(natural science ),Vol.20, No.3 , 7-10

O.L. Mangsarian, Nonlinear Programming (McGraw-Hill, New York, 1969).

S.Bazaraa Mokhtar and C.M.Shetty, Nonlinear programming Theory and 
Algorithm, John Wiley\& Sons New York, 1979.

analysis and applications, 275,251-262 ,2002.

X. Chen, Some Properties of Semi-E-Convex

Functions, journal of mathematical

\section{التحلب الأستقرابي للمجموعة والدالة}

(non- في هذا البحث تم تقديم مفهوم جديد لتقريب التحدب لكل من المجموعات غير المحدبة non-convex ) بالنسبة لعائلة من المجموعات المحدبة وكذلك الدوال غير المحدبة convex sets ) (factions بالنسبة لعائلة من الدو ال المحدبة ـ سمي هذا التقريب للتحدب بالتحدب ألاستقرابي , وتم

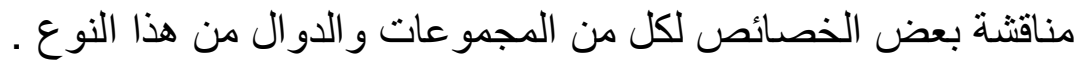

